\title{
Silhouette-based multi-view human action recognition in video
}

\begin{abstract}
In this paper, a human action recognition method is presented where pose features are represented using contour points of the human silhouette, and actions are learned by using sequences of multi-view contour points. The differences and divergences among actors performing the same action are handled by considering variations in shape and speed. Experimental results on the IXMAS dataset show promising success rates, exceeding that of existing multi-view human action recognition state-of-the-art techniques.
\end{abstract}

Keyword: 2D wavelet; C5.0 classifier; Contour points; Human action recognition; IXMAS dataset; Silhouette; Style 